\title{
Calificando al Presidente y percibiendo el aumento del crimen en México Ricardo R. Gómez Vilchis*
}

\section{Resumen}

¿Qué tan importante es la percepción del aumento del crimen cuando el ciudadano califica al Presidente? Este artículo expone a México como un estudio de caso para examinar esta relación. La investigación emplea 11 encuestas nacionales de 1994 a 2006. El estudio analiza los efectos de la percepción del crimen en la calificación que los mexicanos otorgan al Ejecutivo antes y después de la elección del año 2000. La proposición principal es que después de la transición de 2000, la percepción del crimen, junto con la de otras variables económicas, se vuelve más relevante y tiene efectos más fuertes cuando la gente evalúa al Presidente, debido a un incremento de las expectativas de la ciudadanía en torno a la competencia del Ejecutivo.

\begin{abstract}
How important are citizen perceptions of an increase in crime rate when they evaluate the President? This article uses Mexico as a case study to examine the relationship between perception of crime and citizen grading of the President. The research uses 11 national surveys from 1994 to 2006 to analyze the effects of perception of crime on citizen grading of the President before and after the 2000 presidential election. The main proposition is that, after the 2000 political transition, perception of crime, together with other economic variables, becomes more relevant and has stronger effects when citizens evaluate the President due to an increase of their expectations of the Executive's competence.
\end{abstract}

Palabras clave: Alternancia, calificación sobre el Presidente, crimen, democracia, México, transición.

Key words: Political alternation, citizens' grading of the President, crime, democracy, Mexico, transition.

\footnotetext{
Ricardo R. Gómez Vilchis es doctor en Ciencia Política por la Universidad de California, San Diego. Profesor-investigador Universidad de Guanajuato, adscrito al Departamento de Estudios Políticos de la División de Derecho, Política y Gobierno.
} 
Ante los ojos de la ciudadanía, la mayoría de los países latinoamericanos, incluyendo a México, han realizado un trabajo pobre para reducir el crimen. La evidencia muestra que estas naciones han fracasado para erradicar este problema. Según la Oficina sobre Drogas y Crimen de las Naciones Unidas (UNDOC, por sus siglas en inglés, 2011), Honduras, El Salvador, Venezuela y Colombia, entre otros países latinoamericanos, han estado entre las naciones con mayor número de homicidios en el mundo en los últimos años. Al mismo tiempo, el entusiasmo de la opinión pública por el arribo de la democracia ha disminuido porque varios de los "nuevos regímenes" democráticos no han tenido un buen desempeño. Son acusados de ser ineficientes para combatir el crimen y de favorecer solo a los ricos (Mainwaring y Pérez-Liñán, 2005: 53).

Más que una excepción, los altos niveles de criminalidad han sido una constante en América Latina. El nivel de homicidios en El Salvador fue de 138 por cada 100,000 habitantes en 1995 (Wood, 2005: 197). En Colombia la situación no puede considerarse mejor: 80 personas murieron por cada 100,000 habitantes en 1991 (Hagopian, 2005: 349). En Guatemala, el nivel de homicidios alcanzó casi los 60 por cada 100,000 habitantes en 1996 (Seligson, 2005: 226). Estas cifras incluso superan el clima de violencia de los países africanos involucrados en conflictos bélicos. Sudáfrica tuvo poco más de 55 homicidios por cada 100,000 habitantes durante la etapa de mayor conflicto de su guerra civil en 1982 (Hagopian, 2005: 350). Más recientemente, en 2004, la región de América Latina registró uno de los más altos niveles de violencia en el mundo, según la UNODC (2011). El clima de inseguridad ha hecho que la población civil sea altamente vulnerable a la violencia. En México, $60 \%$ de los ciudadanos declararon que ellos o su familia habían sido víctimas de algún tipo de crimen en los últimos 12 meses, en 2003 (Hagopian, 2005: 348).

El presente trabajo utiliza a México como un estudio de caso y busca contestar la siguiente pregunta: ¿de qué manera la percepción sobre el crimen afecta la forma en que los ciudadanos califican a la figura presidencial antes y después de una transición democrática? Este estudio pretende contribuir al entendimiento de un problema común en América Latina: la visión del ciudadano sobre el crimen es una determinante que puede disminuir el apoyo de la opinión pública a las instituciones y a los líderes políticos.

La investigación analiza los efectos que la percepción del crimen tuvo en la calificación que el Presidente recibió de los mexicanos de 1994 a 2006. La proposición principal es que una transición democrática puede aumentar 
las expectativas sobre la competencia del gobierno en turno, en temas de orden político-social, como el crimen, junto con los de índole económica. Los votantes consideran que el nuevo presidente electo debe preocuparse por los principales problemas de la ciudadanía (Cornelius y Craig, 1991: 25).

El análisis se enmarca en el área del comportamiento político y de la sociología política, específicamente en el impacto que los problemas de índole social de gran relevancia tienen en el ciudadano. Se busca examinar de manera empírica de qué forma la percepción del crimen afecta la calificación que otorgan los ciudadanos a las instituciones políticas, en este caso del Poder Ejecutivo. El votante evalúa las instancias de gobierno y las aprueba o las desaprueba según la visión que tiene sobre su entorno social y político.

El presente estudio maneja 11 encuestas nacionales realizadas entre 1994 y 2006. Primero, se examina en la literatura la presencia del crimen como un problema en América Latina. La segunda sección analiza el contexto mexicano después de la alternancia y pone énfasis en el aumento de las expectativas ciudadanas sobre la competencia del Presidente para atender el problema del crimen. La metodología describe cómo los datos fueron obtenidos para probar empíricamente los efectos de la percepción sobre el crimen en la forma en que los ciudadanos califican al Ejecutivo. Posteriormente, se presenta un análisis econométrico y, finalmente, las conclusiones del trabajo.

\section{Aumento del crimen en las emergentes democracias latinoamericanas}

La evidencia empírica indica que la mayoría de los gobiernos en América Latina ha fracasado en el propósito de reducir el crimen. En estas democracias emergentes cada vez es menor la tolerancia de la ciudadanía ante la falta de eficacia de los mandatarios para reducir el problema de la inseguridad, lo cual ha afectado una eventual consolidación del sistema democrático. Los análisis muestran que la ciudadanía asocia fuertemente las demandas de tipo social, en particular el combate contra la inseguridad, con las obligaciones que tiene un régimen democrático (Moreno, 2001: 43). Algunos ejemplos así lo muestran: 38\% de los costarricenses percibe la lucha contra el crimen como la principal tarea que debe atender un gobierno democrático (Clark, 2001: 87); en Chile la cifra es de 26\% y en México de 31\% (Klesner, 2001: 125). 
Un clima de inseguridad, caracterizado por altos niveles de criminalidad, puede ser un elemento clave que impida la consolidación democrática a través del fortalecimiento indirecto de valores autoritarios y de desconfianza hacia la democracia. Bermeo (1999) argumenta que el desorden social derivado de la inseguridad y el crimen es un factor significativo que conduce a los votantes a apoyar regímenes autoritarios y, por lo tanto, es la principal razón de la caída de los sistemas democráticos. La base de datos de Bermeo (1999) incluye países que han sufrido algún retroceso democrático, y muestra que aquellas naciones cuyo nivel de criminalidad promedió siete homicidios por cada 100,000 habitantes experimentaron algún retorno hacia el autoritarismo. Si se toma este dato como referente, las probabilidades de la consolidación democrática en América Latina son escasas. Se ha estimado que el número de homicidios por cada 100,000 habitantes en Latinoamérica es de 30 (Seligson, 2005: 225). Sin embargo, esta cifra puede ser rebasada por varias naciones del área (El Salvador, Colombia y Guatemala, entre otras) como se mencionó al principio de este artículo.

Lo anterior ha favorecido el fortalecimiento de una cultura autoritaria entre los habitantes de la región. En 1999 60\% de los guatemaltecos prefirió un gobierno de mano dura por encima de uno que motivara la participación ciudadana como principal opción para resolver los problemas del país (Seligson, 2005: 227). En ese mismo año en El Salvador, 55\% de la población manifestó que un golpe de Estado podía ser justificado en un clima de desorden y crimen (Hagopian, 2005: 350). También en 1999, en México la mitad de la población apoyaba un gobierno de tipo autoritario. Además, sólo uno de cada tres mexicanos expresó sentirse satisfecho con la democracia (Camp, 1999: 232).

En este artículo el crimen es entendido como un acto o bien omisión que la ley marca como punibles. Puede ser también alguna violación al marco legal, la cual es considerada la causa de una acción criminal. La UNODC (2011) centra su análisis y definición en el número de homicidios que se cometen en un país por cada 100,000 habitantes.

\section{Contexto político y expectativas en torno a las instituciones y sus líderes: el caso mexicano como ejemplo}

Edwards, Mitchell y Welch (1995: 109) sostienen que un issue (tema económico o político de trascendencia) sólo puede afectar las preferencias de la opinión pública si éste es relevante para el votante. En otras palabras, el contexto puede incrementar o disminuir el potencial de ciertos temas 
y afectar la forma en que la opinión pública evalúa, califica y genera expectativas en torno a las instituciones políticas y los líderes que las dirigen. Esta sección describe y explica algunos casos que respaldan el argumento de estos autores, enfatizando el caso mexicano en la elección del año 2000.

En Perú, el aumento de la violencia derivada de la guerrilla no afecta de igual forma el apoyo popular a los líderes políticos de la derecha que a los de izquierda. Cuando la violencia de grupos como Sendero Luminoso se hacía más evidente para el electorado durante la administración de Alan García (1985-1990), un presidente de izquierda, la ciudadanía lo castigaba con una baja aprobación porque consideraba que el Ejecutivo era débil para encarar el problema, lo cual no ocurría con un gobierno de derecha (Arce, 2003: 572). ${ }^{1}$ En la Rusia poscomunista no sólo las variables económicas son claves para entender el comportamiento del votante. La percepción ciudadana sobre los atributos de los políticos como la fortaleza, el liderazgo de "mano firme" y la ideología nacionalista es central para entender quién será favorecido con el voto. El votante refleja en su comportamiento político el deseo de un ejercicio del poder hasta cierto punto autoritario, por parte de quienes gobiernan (Mishler y Willerton, 2003: 111). ${ }^{2}$ Algo similar ocurre en China, donde el ciudadano tiene una marcada preferencia por los gobiernos que privilegian sociedades donde impera el orden, incluso a costa de limitar las garantías individuales. Frente a la disyuntiva de un gobierno que proteja las libertades civiles e individuales o un sistema que garantice el control y el orden, los votantes chinos favorecen la segunda opción, y cada vez que lo hacen las probabilidades de aprobar a las autoridades en turno aumentan significativamente (Zhong, Chen y Scheb, 1998). En contextos tan disímbolos como Perú, Rusia y China, la opinión pública tiene algo en común que "marca" su comportamiento político: las expectativas del ciudadano sobre las instituciones y los líderes políticos no vienen de un vacío; el contexto político restringe las expectativas que los ciudadanos depositan en sus gobernantes. En el caso de México, la pregunta sería entonces la siguiente: ¿de qué forma el contexto imperante en el país afecta

1 Cuando Fujimori (1990-1997) estaba en el poder y los votantes percibían un aumento de la violencia derivado de la lucha contra la guerrilla, esta variable no afectaba la aprobación presidencial porque la gente consideraba que al ser un gobierno de derecha el que encaraba el problema de la guerrilla, resultaba natural que la "mano dura" del presidente despertara, en primera instancia, una reacción de los grupos guerrilleros, quienes intensificaban la violencia en el país (Arce, 2003: 573).

2 En la Rusia de Yeltsin y Putin cuando los ciudadanos aprobaban la suspensión del Parlamento, del sistema de partidos o cuando consideraban que un uso dictatorial del ejercicio del poder era la única forma de "encarar" situaciones de crisis, las probabilidades de aprobar al presidente en turno se incrementaban significativamente (Mishler y Willerton, 2003: 112). 
las expectativas que la ciudadanía tiene sobre las instituciones políticas y los líderes que las dirigen, en específico el Presidente?

Entender el cambio que generó la elección de 2000 en la percepción de la opinión pública en México es fundamental para comprender la forma en que el contexto político afecta las expectativas que la ciudadanía tiene en relación con la competencia de los dirigentes de las instituciones políticas, quienes afrontan los temas de la agenda nacional. Como muestra la Gráfica 1, en febrero de 2000, a unos meses de la elección presidencial de ese año, solo $40 \%$ de la población consideró que México era una democracia; en julio de 2000, unos cuantos días después del proceso electoral, 63\% de los mexicanos pensaba que se vivía en un sistema democrático (Camp, 2004: 39).

\section{Gráfica 1: Sentir ciudadano sobre si México es una democracia, antes y después de la elección de $\mathbf{2 0 0 0}$}

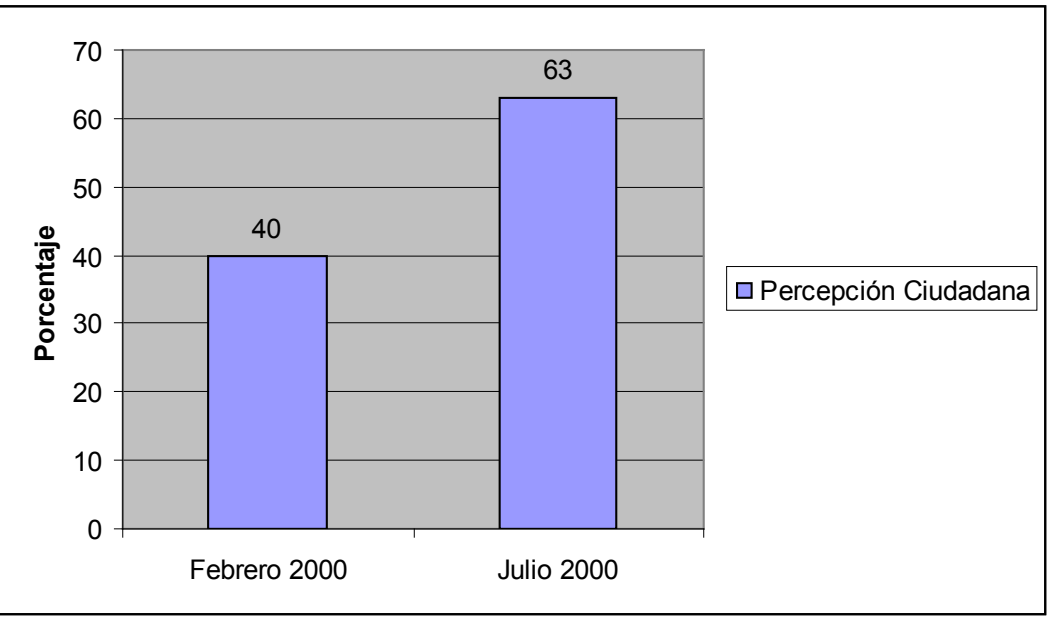

Fuente: (Camp, 2004: 39). La pregunta que se formuló fue: “¿Es México una democracia?”. Primero a cinco meses antes de la contienda electoral y después a sólo unos días de haber pasado la elección presidencial de 2000.

Para Magaloni y Poiré la elección en 2000 de Vicente Fox como Presidente de México fue la expresión de un deseo por el cambio (2004). No de un cambio por profundas reformas estructurales, sino de uno basado principalmente en las expectativas de una mayor competencia del Presidente electo en un mejor manejo de la economía y la lucha contra el crimen (Magaloni y Poiré, 2004: 315). Estos autores encuentran que la opinión del votante sobre temas como el control de la economía, la política y la lucha contra el 
crimen (problemas asociados con las expectativas ciudadanas por elegir un nuevo presidente con mayor competencia) fueron variables altamente significativas en la elección de 2000 (Magaloni y Poiré, 2004: 307). ${ }^{3}$

Se esperaba que el nuevo presidente resolviera los asuntos más urgentes y relevantes del país como la lucha contra el crimen. De hecho, como muestra la Gráfica 2 (véase en la siguiente página), los niveles de confianza de Fox durante su primer año de gobierno son notablemente más altos (63\%) que los de Salinas y Zedillo (44\% y 33\%, respectivamente). ${ }^{4}$ De esta manera, es posible suponer que después de la elección de 2000, el vínculo entre la forma en que los votantes calificaban al Ejecutivo y la percepción de la ciudadanía sobre temas político-sociales como el crimen y de índole económica se hizo más fuerte debido a las altas expectativas por parte de los votantes sobre la pericia del Presidente para encarar los problemas.

\section{Gráfica 2: Niveles de confianza en tres presidentes mexicanos después de su primer año de gobierno}
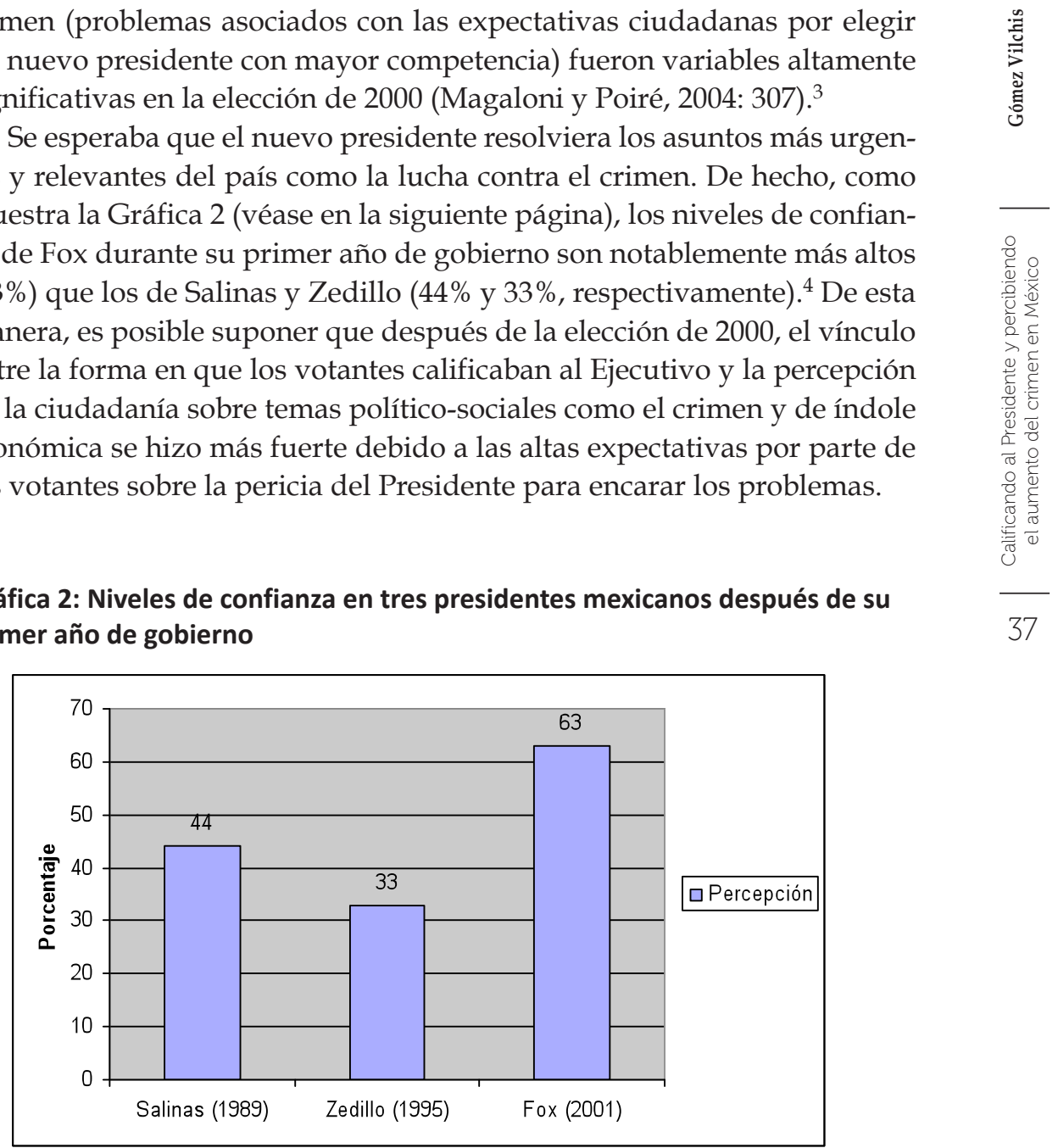

Fuente: BIIACS, www.biiacs.cide.edu.

La pregunta fue: "Cuando el Presidente anuncia una nueva política pública, ¿usted le cree?".

3 Cada vez que un votante consideraba que el crimen era el "tema" más importante para la nación, las probabilidades de apoyar a Francisco Labastida, candidato del Partido Revolucionario Institucional (PRI), disminuían en $17 \%$; en casi $18 \%$ cuando pensaba que la política era el principal reto a resolver y en casi 15\% cuando la economía era la principal preocupación del votante.

El autor hizo la gráfica con los datos obtenidos de las encuestas del Banco de Información para la Investigación Aplicada en Ciencias Sociales, BIIACS (1989, 1995 y 2001), citadas en la bibliografía. En el caso de 1995, se usaron dos encuestas y se obtuvo el promedio del nivel de confianza de la ciudadanía en el Presidente. En 2001, se usaron tres y se promediaron. 
Una elección competitiva, como fue la de 2000, y una eventual transición incitaron a los ciudadanos a asociar la democracia con la reducción del crimen. Antes de la elección de 2000 en México, 31\% de la gente consideraba que el crimen era una tarea sustancial para la democracia (Klesner, 2001: 125). Esta asociación entre la democracia y la lucha contra el crimen en la mente de los ciudadanos se fortaleció después de la alternancia. Durante su gestión, Vicente Fox, primer presidente proveniente del Partido Acción Nacional (PAN), de cara a la opinión pública hizo del combate contra el crimen una prioridad de su administración. El nuevo presidente manifestó que los altos niveles de crimen en el país se reducirían. Durante la administración foxista se creó la Secretaría de Seguridad Pública (SSP) y la Policía Federal Preventiva (PFP) fue adscrita a esa dependencia. Asimismo, la Policía Judicial Federal, dependiente de la Procuraduría General de la República (PGR), se transformó en la Agencia Federal de Investigaciones (AFI), en lo que se presentó como un modelo de policía investigadora basada en las experiencias de la Agencia Federal de Investigación (FBI, por sus siglas en inglés) y la Scotland Yard (Sánchez, 2011: 110).

La mayoría de los anteriores estudios ha enfatizado el papel que la percepción de la economía juega cuando los votantes asignan una calificación al Ejecutivo (Buendía, 1996: 567; Domínguez y McCann, 1995: 34; Magaloni, 2006: 82; Moreno, 2004: 9 y Villarreal, 1999: 132). Sin embargo, poco o nada se sabe en México sobre los efectos de la percepción del crimen en la manera en que los ciudadanos ven al Presidente. El presente análisis se enfoca en este país y prueba empíricamente los efectos que la percepción sobre este problema, un tema relevante en América Latina, tiene en la manera en que el primer mandatario es calificado por la ciudadanía.

\section{Hipótesis}

La transición de 2000 fue un "suceso político relevante" que modificó la manera como los ciudadanos veían al régimen político. En párrafos anteriores se mostró que a unos meses de la elección presidencial de ese año, en febrero, sólo $40 \%$ de la población consideró que México era una democracia; en julio de 2000, pocos días después de la elección, el porcentaje aumentó a 63\% (Camp, 2004: 39).

Asimismo, es probable que se haya alterado la forma como los ciudadanos veían al Presidente. Los votantes empezaron a mirar al Ejecutivo de manera distinta y tuvieron mayores expectativas sobre la competencia del nuevo mandatario para manejar la economía y problemas de tipo político- 
social como el crimen. Teniendo en cuenta lo anterior, el análisis parte de las siguientes hipótesis:

H 1: Manteniendo las otras variables constantes, los efectos de la percepción de los ciudadanos sobre el crimen en la calificación que le otorgan al Presidente deben ser más fuertes después de la transición de 2000 que durante el dominio priísta anterior a ésta.

H 2: Manteniendo las otras variables constantes, los efectos de la percepción de la ciudadanía sobre la economía en la calificación que dan al Presidente deben ser más fuertes después de la transición de 2000 que durante el dominio priísta anterior a ésta.

\section{Datos y metodología}

El presente estudio descansa en la evidencia que proviene de encuestas nacionales realizadas en México. ${ }^{5}$ Las 11 encuestas nacionales en las que se basa este estudio fueron hechas por Parametría y la Oficina de la Presidencia (OP). El número de observaciones por cada encuesta oscila entre 3,000 y 600 entrevistados. ${ }^{6}$ En el caso de la OP, las encuestas fueron financiadas a través

5 Este país fue escogido por múltiples razones. Primero, el caso permite examinar cómo la ciudadanía evalúa al Presidente antes y después de la transición de 2000. Debido a que los gobiernos no democráticos intentan controlar el flujo de la información política, éstos raramente permiten el libre acceso del público a los estudios de opinión realizados por medio de encuestas. Sin embargo, la disponibilidad de los datos que se tienen permite estudiar la calificación de la ciudadanía sobre el Ejecutivo durante los últimos años del PRI en el poder, específicamente de 1994, 1995 y 2000. Desafortunadamente, no se tienen observaciones para cada año del presente estudio, es decir, de 1994 a 2006. Durante el régimen de Salinas y Zedillo no se preguntó regularmente a la ciudadanía su percepción sobre la economía cuando se le solicitaba su opinión sobre el crimen. Por esta razón no se pudo incluir este tipo de datos. En el caso del periodo de Fox, en 2005 no se preguntó a los votantes sobre su visión del crimen en México. Asimismo, preguntas que permitieran medir los efectos fijos por el Presidente (atributos, personalidad, carisma) no fueron incluidas regularmente en las encuestas, por lo que tampoco pudieron ser tenidas en cuenta en el análisis econométrico. La segunda razón para estudiar el caso mexicano es que después de la alternancia, el nuevo gobierno ha estado en el poder durante un periodo estable. Esta condición ofrece la oportunidad de estudiar la percepción de la ciudadanía en un periodo más o menos largo. Finalmente, la transición en sí misma y los datos obtenidos por el investigador durante los últimos cinco años permiten el uso de la elección de 2000 para examinar empíricamente los efectos de la percepción del crimen en la forma que los ciudadanos califican al Presidente antes y después de una alternancia pro-democrática.

6 La encuesta de 1994 tuvo 3,973 observaciones; la de 1995 alcanzó 2,558; la de 2000, 600; la de marzo de 2001 llegó a 1,141; la de mayo de ese año fue de 1,068; la de noviembre registró 1,189; la de 2002 incluyó 1,234 entrevistados; la de 2003, un total de 1,121; la de 2004, la cifra de 1,175; la de septiembre de 2006 obtuvo 1,010; la de noviembre de ese año, 1,000. 
del presupuesto federal y son confidenciales y para uso gubernamental. Sin embargo, la Asesoría Técnica de la Presidencial de la República las hizo públicas después de que Salinas (88-94) y Zedillo (94-00) terminaron sus administraciones. Hoy estas bases de datos son públicas y están disponibles en el BIIACS (2011), www.biiacs.cide.edu. ${ }^{7}$

En total las encuestas contienen cerca de 16,000 entrevistas hechas en viviendas y 600 vía telefónica entre noviembre de 1994 y noviembre de 2006. Ese periodo cubre tres diferentes administraciones (Carlos Salinas de Gortari, Ernesto Zedillo, ambos priístas, y el panista Vicente Fox). Hay una encuesta durante el gobierno de Salinas (en 1994); dos durante la administración de Zedillo (en 1995 y 2000), todas organizadas por Asesoría Técnica de la Presidencial de la República. Hay ocho encuestas que cubren el gobierno de Fox, seis conducidas por la Asesoría Técnica de la Presidencial de la República (2001, 2002, 2003, 2004), y dos por Parametría en 2006. Tres encuestas se hicieron en 2001.

Cuando las encuestas fueron hechas por la OP, las viviendas fueron localizadas a través de un proceso multietápico, estratificado y probabilístico. En cada hogar se llevó a cabo una entrevista de aproximadamente 40 minutos con un adulto, escogido al azar, con una proporción aproximadamente igual entre hombres y mujeres. Cuando Parametría organizó las encuestas, las viviendas se ubicaron a través de un proceso multietápico no estratificado y probabilístico. En cada casa se realizó una entrevista de aproximadamente 25 minutos con un encuestado, seleccionado al azar.

El análisis reconoce la presencia de preguntas categóricas, como la identificación partidista y el género, entre otras, así como el hecho de que la existencia de preguntas referidas a la calificación sobre el Presidente, las percepciones ciudadanas en torno al crimen y los temas económicos “generan" respuestas numéricas ordenadas (Baum, 2006: 161). A las respuestas de estas preguntas se les da un manejo cuantitativo, asumiendo la cardinalidad en la codificación de las mismas. El estudio pudo haber usado modelos ordinales de tipo logit binomial, por ejemplo; sin embargo, optó por "tratar" a las escalas de las respuestas categóricas y ordinales como variables cardinales, ya que al elegir modelos OLS (Ordinary Least-Squares Regression, por sus siglas en inglés, mínimos cuadrados ordinarios en es-

7 Cuando los cuestionarios fueron diseñados por la OP, las encuestas fueron conducidas por Opinión Profesional, S.A. de C.V., una agencia privada especializada en encuestas y grupos focales. Los trabajadores de campo no fueron informados de la identidad del cliente. Se identificaron ante los encuestados solo como empleados de Opinión Profesional. 
pañol), se podía capturar una mayor "variación" de las respuestas ciudadanas sobre la calificación del Presidente.

El presente trabajo se basa en un análisis que parte de un modelo MLC (lineal clásico), el cual permite calcular el efecto marginal de las variables independientes sobre la dependiente (qué tanto cambia Y por el incremento de una unidad en X) (Wooldridge, 2001: 32). La variable dependiente es la calificación que los ciudadanos otorgan al Presidente, con base en una escala que va de uno a cinco, donde uno es la calificación más baja y cinco la máxima. La pregunta: "En una escala del 1 al 5, ¿cómo calificaría la actuación del Presidente?" permite "medir" el impacto, a diferentes niveles, que tiene la percepción de los ciudadanos en torno al crimen sobre la calificación que le otorgan al Ejecutivo. El Cuadro 1 presenta la operacionalización y la codificación de las variables. La variable independiente clave es la percepción sobre el crimen. El análisis también incluye como controles una serie de variables, principalmente relacionadas con la percepción ciudadana sobre los temas económicos, que han sido utilizadas en investigaciones previas (Domínguez y McCann 1995, 39; Magaloni 2006, 165; Moreno 2006, 35; 2009, 279; Villarreal 1999, 136).

Se espera que cuando los ciudadanos piensan que el crimen aumenta, el Presidente recibirá una baja calificación; esta variable tendrá mayores efectos en la calificación que los ciudadanos le dan al Ejecutivo después de la transición. Las percepciones sobre el aumento del crimen están correlacionadas negativamente con la evaluación del Presidente. Las expectativas sobre el desempleo y la inflación son similares a aquellas que los votantes tienen sobre el crimen. La gente castiga al Presidente con una baja calificación cuando cree que el desempleo y la inflación aumentan. El estudio predice que cuando los encuestados piensan que la economía marcha bien, el Presidente obtendrá una buena calificación. La correlación aquí es positiva. El análisis anticipa que la identificación partidista tiene un efecto positivo en la calificación del Presidente, ya que el ciudadano tiende apoyar a los políticos que pertenecen al partido con el cual se identifica. El estudio incluye indicadores sociodemográficos - ingreso, edad, educación y género - como variables control. El grupo referente para ingreso es la clase baja; para edad, los jóvenes de 18 a 25 años; para la educación, la gente sin preparación escolarizada; para el género, las mujeres. Debido a la codificación original de algunas encuestas, que no daban muchas opciones de respuesta, se tuvo que organizar como una sola variable a la clase media y la clase alta. 
Cuadro 1. Explicación de la aprobación presidencial

\begin{tabular}{|c|c|c|}
\hline Concepto & Pregunta & Operacionalización y codificación \\
\hline $\begin{array}{l}\text { Variable dependiente: } \\
\text { Calificación sobre el Presidente }\end{array}$ & $\begin{array}{c}\text { ¿Cómo calificaría la actuación hasta } \\
\text { estos momentos de (Salinas, Zedillo, } \\
\text { Fox) como Presidente } \\
\text { de la República? }\end{array}$ & $\begin{array}{l}\text { Se utiliza una escala que va de } 1 \\
\text { a } 5 \text {, donde } 1 \text { es la peor } \\
\text { calificación y } 5 \text { la mejor. }\end{array}$ \\
\hline \multicolumn{3}{|l|}{ Variables independientes: } \\
\hline $\begin{array}{l}\text { Percepción del aumento } \\
\text { del crimen }\end{array}$ & $\begin{array}{l}\text { Desde que Salinas (Zedillo o Fox) es } \\
\text { presidente, ¿usted considera que el } \\
\text { crimen ha aumentado o disminuido } \\
\text { en México? }\end{array}$ & $\begin{array}{l}\text { (0) Ha disminuido } \\
\text { (1) Sigue igual } \\
\text { (2) Ha aumentado }\end{array}$ \\
\hline $\begin{array}{l}\text { Percepción del aumento } \\
\text { del desempleo }\end{array}$ & $\begin{array}{l}\text { Desde que Salinas (Zedillo o Fox) } \\
\text { es presidente, ¿usted considera } \\
\text { que el desempleo ha aumentado o } \\
\text { disminuido en México? }\end{array}$ & $\begin{array}{l}\text { (0)Ha disminuido } \\
\text { (1)Sigue igual } \\
\text { (2)Ha aumentado }\end{array}$ \\
\hline $\begin{array}{l}\text { Percepción del aumento } \\
\text { de la inflación }\end{array}$ & $\begin{array}{l}\text { Desde que Salinas (Zedillo o Fox) es } \\
\text { presidente, ¿̇usted considera que la } \\
\text { inflación ha aumentado o disminuido } \\
\text { en México? }\end{array}$ & $\begin{array}{l}\text { (0)Ha disminuido } \\
\text { (1)Sigue igual } \\
\text { (2)Ha aumentado }\end{array}$ \\
\hline $\begin{array}{l}\text { Percepción de la situación } \\
\text { económica del país }\end{array}$ & $\begin{array}{l}\text { Comparada con el año anterior, ¿̇la } \\
\text { situación económica actual del país } \\
\text { es...? }\end{array}$ & $\begin{array}{l}\text { (1) Peor } \\
\text { (2) La misma } \\
\text { (3) Mejor }\end{array}$ \\
\hline $\begin{array}{l}\text { Percepción de su situación } \\
\text { económica personal }\end{array}$ & $\begin{array}{l}\text { ¿Usted piensa que su situación } \\
\text { económica personal es mejor, igual o } \\
\text { peor que hace un año? }\end{array}$ & $\begin{array}{l}\text { (1) Peor } \\
\text { (2) La misma } \\
\text { (3) Mejor }\end{array}$ \\
\hline
\end{tabular}

Identificación partidista

¿Usted se considera panista, priísta o perredista?

¿Cuál es su ingreso familiar?

¿Cuántos años tiene?

¿Cuál es su nivel de escolaridad?

Marque su género
Variable binaria (1) Si el votante simpatiza con el partido del Presidente; (0) si éste no es el caso. De 1994 a 2000, (1) si el votante era prí́sta. De 2001 a 2006 (1), si el votante era panista

(1) Clase baja, de 0 a 1 salario mínimo

(2) Clase media baja, de más de 1 salario mínimo a 3 salarios mínimos (3) Clase media y clase alta, más de 3 salarios mínimos

(1) Jóvenes, de 18 a 25 años (2) Adultos jóvenes, de 26 a 40 años

(3) Adultos, de 41 a 60 años

(4) Adultos mayores, de 61 años en adelante

\begin{tabular}{c|cl} 
Educación & ¿Cuál es su nivel de escolaridad? & (1) Ninguno \\
& (2) Primaria \\
& (3) Secundaria \\
& (4) Preparatoria \\
& (5) Licenciatura o posgrado \\
& (0) Mujer \\
& (1) Hombre \\
\hline PRI & (0)Después de la era del PRI, \\
& de 2001 a 2006 \\
& (1) Durante el dominio del PRI, \\
& de 1994 a 2000 \\
\hline
\end{tabular}


Cuadro 2: Describiendo las variables claves (promedio)

\begin{tabular}{ccc}
\hline Variables & $1994-2000$ & $2001-2006$ \\
\hline Calificando al Presidente & 3.71 & 3,61 \\
& $(1.11)$ & $(1,09)$ \\
Percibiendo el aumento del crimen & .50 & .90 \\
Percibiendo aumento del desempleo & $(.43)$ & $(.77)$ \\
& .84 & .89 \\
Percibiendo aumento de la inflación & $(.79)$ & $(.80)$ \\
& .95 & .85 \\
Percepción economía del país & $(.75)$ & $(.78)$ \\
& 81 & .97 \\
Percepción económica personal & $(.80)$ & $(.71)$ \\
& .92 & .94 \\
Identificación partidista & $(.86)$ & $(.70)$ \\
& .45 & .30 \\
\end{tabular}

Nota: Desviación estándar en paréntesis.

\section{Resultados}

Como parte del análisis, este estudio presenta en el Cuadro 2, la estadística descriptiva básica de las principales variables explicativas. Estos datos muestran que en ambos periodos, de 1994 a 2000, y de 2001 a 2006, los presidentes mexicanos (Salinas, Zedillo y Fox) fueron calificados de forma positiva por la ciudadanía. La ciudadanía apoya el trabajo del Ejecutivo antes y después de la alternancia. La diferencia entre estos dos periodos es mínima. Por otro lado, la percepción del aumento del crimen es mayor durante el periodo pos-PRI. En cambio, la percepción del aumento de la inflación resultó mayor en la etapa priísta. Las diferencias de las restantes variables en un periodo y en el otro son más bien mínimas, salvo en el caso de la percepción económica del país y de la identidad partidista. En el primero, el promedio es mayor después de la alternancia; en el segundo, antes de la misma.

El Cuadro 3 muestra que hay diferencias significativas en cómo la ciudadanía califica a los presidentes priístas (Salinas y Zedillo) y a un Ejecutivo que proviene de la oposición, Fox. Durante la etapa predemocrática, el Modelo 1 indica que cuando la gente piensa que el crimen aumenta, la calificación que recibe el Presidente, materialmente, no sufre cambio alguno. Esta variable 


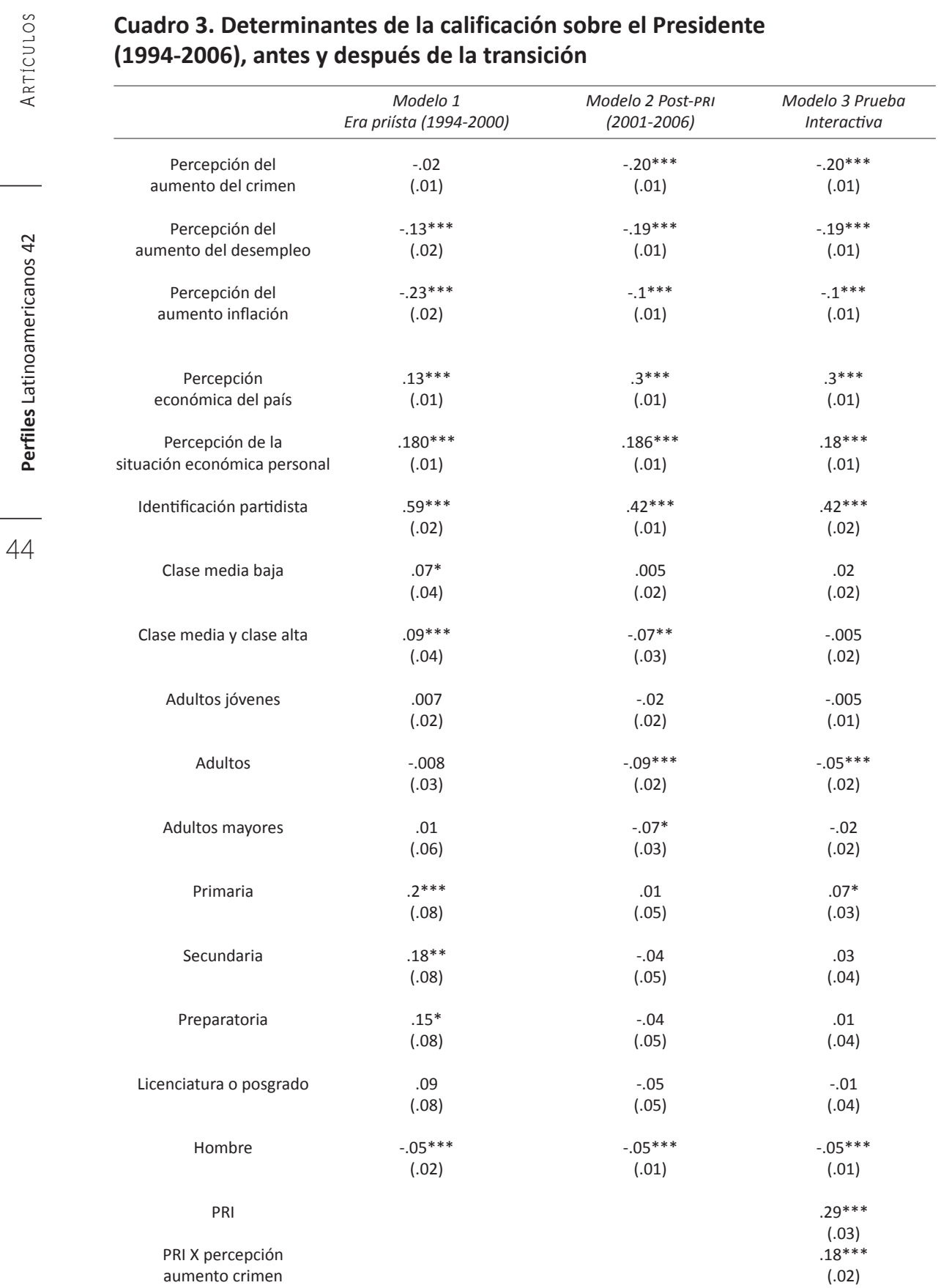


Cuadro 3. (Continuación.)

\begin{tabular}{|c|c|c|c|}
\hline & $\begin{array}{c}\text { Modelo } 1 \\
\text { Era priista (1994-2000) }\end{array}$ & $\begin{array}{c}\text { Modelo } 2 \text { Post-PRI } \\
\text { (2001-2006) }\end{array}$ & $\begin{array}{l}\text { Modelo } 3 \text { Pruebo } \\
\text { Interactiva }\end{array}$ \\
\hline $\begin{array}{l}\text { PRI X percepción } \\
\text { aumento desempleo }\end{array}$ & & & $\begin{array}{l}.05^{* *} \\
(.02)\end{array}$ \\
\hline $\begin{array}{l}\text { PRI X percepción } \\
\text { aumento inflación }\end{array}$ & & & $\begin{array}{c}-.12 * * * \\
(.02)\end{array}$ \\
\hline $\begin{array}{l}\text { PRI X percepción } \\
\text { económica del país }\end{array}$ & & & $\begin{array}{c}-.17^{* * *} \\
(.02)\end{array}$ \\
\hline $\begin{array}{c}\text { PRI X percepción } \\
\text { situación económica personal }\end{array}$ & & & $\begin{array}{l}.001 \\
(.02)\end{array}$ \\
\hline $\begin{array}{l}\text { PRI X identificación } \\
\text { partidista }\end{array}$ & & & $\begin{array}{l}.15^{* * *} \\
(.03)\end{array}$ \\
\hline $\mathrm{N}$ & 7,131 & 8,938 & 16,069 \\
\hline $\mathrm{R}^{2}$ & .34 & .37 & .36 \\
\hline
\end{tabular}

Nota: Error estándar en paréntesis. La variable dependiente es la calificación sobre el Presidente ${ }^{*} p<.10,{ }^{*} p<.05$,

$* * * p<.01$. Son modelos lineales clásicos. El análisis incluye variables binarias por cada año, excepto por uno de ellos en la estimación de los modelos, pero no son presentadas en los cuadros. La mayoría de estas variables no son significativas. Se realizó el Breusch-Pagan/Cook-Weisberg Version of test para identificar algún problema de heterocedasticidad, el cual fue corregido corriendo regresiones usando "errores estándar robustos". El nivel del factor inflacionario de varianza (variance inflation factor) en todos los modelos reportó ser un número bastante pequeño, menor a cuatro, que es el umbral que se toma para saber si hay algún problema de multicolinealidad.

no es significativa en términos estadísticos. En contraste con los últimos años de la etapa priísta, el Modelo 2 ilustra que la percepción del crimen tiene fuertes y significativos efectos, con 99\% de nivel de confianza, cuando el votante evalúa al Presidente. Si la percepción del crimen aumenta una unidad, la calificación de la ciudadanía en torno al Presidente disminuye.

El Modelo 3 prueba empíricamente la diferencia hipotética entre estos dos periodos: los últimos años del PRI y la etapa prodemocrática después de la alternancia de 2000, por medio de la creación de un conjunto de variables interactivas, producto de la variable PRI multiplicada, respectivamente, por cada una de la variables clave del modelo. Cada vez que una de estas variables interactivas es significativa en términos estadísticos, se comprueba que una de estas variables claves tiene diferentes efectos después de la alternancia. Éste es el caso de las variables que miden la percepción sobre el crimen, el desempleo, la inflación, la economía del país y la identificación partidista.

La variable interactiva PRI $x$ Percepción del aumento del crimen del Modelo 3 permite observar que la diferencia entre estos dos periodos (antes y después de la transición) es estadísticamente significativa, con 99\% de nivel de confianza. Este hallazgo muestra que el contexto político afec- 
ta la forma en que el ciudadano evalúa a las instituciones, en este caso al Ejecutivo. Como predijo la hipótesis de investigación uno, antes de la alternancia, durante los últimos años de la etapa priísta, la gente no consideraba que combatir al crimen era una de las principales tareas del Presidente. En cambio, después de la transición el ciudadano comienza a pensar que reducir los índices de criminalidad tiene que ser una tarea obligatoria del gobierno en turno. Debido a esto, la percepción del crimen tiene mayores efectos sobre la calificación que la ciudadanía otorga al Presidente después de la transición de 2000. Se podría contraargumentar que dicho cambio en la percepción de los ciudadanos obedece a un incremento real en los niveles del crimen en México que pudieran coincidir con la alternancia. El argumento anterior no rechaza la hipótesis presentada en el trabajo, ya que ambas causales, el aumento real del crimen y un incremento en las expectativas de la ciudadanía en torno a la competencia del Ejecutivo, podrían explicar el mayor efecto que la percepción del crimen tiene en la calificación otorgada al Presidente. ${ }^{8}$

Incluso, contrario a lo que podría pensarse, los niveles de "criminalidad" (indicadores como el número de homicidios por cada 100,000 habitantes) son más bajos de 2001 a 2006, que de 1994 a 2000, los periodos analizados en este estudio. En 1994 el número fue de aproximadamente 18 homicidios; en 2006, cercano a ocho (Escalante, 2011: 36). Esto fortalece la idea de que la alternancia política provocó que la ciudadanía tuviera más expectativas sobre la determinación del Presidente de reducir el crimen en México y que por tal razón la percepción del votante en torno a tal problema afectara de manera más intensa la calificación ciudadana sobre el trabajo del Ejecutivo, ya que a pesar de que los niveles de criminalidad después de la transición, de 2001 a 2006, son más bajos que de 1994 a 2000, la percepción del crimen tiene mayores efectos sobre la calificación del Presidente después de la alternancia.

Caso distinto es el de la inflación. Como se describe en las siguientes líneas: los mayores niveles de inflación coinciden con un mayor impacto de la percepción de la gente de este indicador económico sobre la variable

8 De igual forma, el presente artículo reconoce sus limitantes metodológicas: en términos estadísticos resulta complicado incluir los niveles del crimen a nivel agregado (de la nación) en un estudio que utiliza datos a nivel de las preferencias individuales del votante, como es el caso de esta investigación, ya que al no contar con la calificación que los ciudadanos otorgan al Presidente cuatrimestral o semestralmente, la variación que ofrece el dato anual (el cual utiliza el presente trabajo) sería muy pobre para poder presentar algún efecto sobre la variable dependiente. 
dependiente antes de 2000. En 1995, durante la crisis económica, en diciembre, la inflación alcanzó el nivel de casi 52\% (INEGI, 2012).

Cuando se describió el Cuadro 2, donde el trabajo presentó cierta estadística descriptiva de las variables claves, se observó que, en promedio, de 2001 a 2006 es mayor el número de ciudadanos que percibe un aumento del crimen en el país, que de 1994 a 2000. Este dato permite encontrar una interpretación alternativa a los hallazgos presentados hasta ahora. La relación entre algunas de las variables explicativas y la dependiente no es lineal. El efecto marginal de X sobre $Y$ no es constante, y depende del valor que tiene $X$. Éste es el caso, probablemente, de la variable percepción del crimen: la percepción del aumento del crimen influye poco cuando es baja (de 1994 a 2000) e influye mucho cuando es alta (de 2001 a 2006). Aunque se puede argumentar que esta explicación no es incompatible del todo con la anterior, el contexto político derivado de la alternancia hizo que la variable percepción del crimen tuviera un mayor impacto al momento de calificar al Ejecutivo, debido a que, precisamente, las observaciones después de la "era del PRI" sobre el aumento del crimen fueron más altas porque las expectativas en torno al Presidente aumentaron como resultado de la alternancia política de 2000.

Si es cierto el argumento de que después de la transición de 2000 el votante tiene mayores expectativas sobre la competencia del Ejecutivo para atender las demandas del ciudadano, podría esperarse que junto con el crimen, las otras variables, vinculadas con los grandes temas del país, o al menos algunas de ellas, tendrían un mayor efecto en la calificación ciudadana del Presidente después de la elección de 2000.

Las variables interactivas del Modelo 3 muestran que los efectos de las percepciones del desempleo que poseen los votantes (la variable PRI x Percepción del aumento del desempleo) y el estado de la economía del país (la variable PRI X Percepción económica del país) son estadísticamente diferentes en los dos periodos de análisis, es decir, tienen un mayor efecto después de la transición. Si bien es cierto que ambas variables tiene un nivel de confianza en ambos periodos, antes y después de la elección de 2000, de un 99\%, el tamaño de los coeficientes es mayor después de la alternancia, indicando que el ciudadano le da mayor relevancia a estos temas a partir de este momento. De esta manera, la percepción del crimen no es la única variable que después de la elección de 2000 tiene mayor efecto en la calificación que los ciudadanos le otorgan al Presidente. Asimismo, es la percepción del crimen la que experimenta el cambio más dramático en términos estadísticos, ya que de no tener significancia alguna en el Modelo 1, alcanza un nivel de confianza de 99\% en el Modelo 2. El hallazgo sugie- 
re que las expectativas de la ciudadanía sobre un nuevo presidente electo, como resultado de una transición, se concentran en el ámbito de lo político y lo social, como es el tema del crimen. De hecho, esto coincide con los hallazgos de investigaciones anteriores, los cuales han mostrado que la gente considera que las tareas sustanciales de la democracia se vinculan con temas políticos, como la lucha contra el crimen, más que con temas económicos (Moreno, 2001: 43; Klesner, 2001: 127).

Si bien es cierto que los resultados de la percepción del desempleo y el estado de la economía del país fortalecen la segunda hipótesis del presente trabajo (los efectos de la percepción de la economía que tienen los ciudadanos en la calificación que otorgan al Presidente, son mayores después de la transición) no ocurre lo mismo con la percepción en torno a la inflación y con la perspectiva del votante sobre el estado de su economía personal. La percepción sobre la inflación tiene mayores efectos antes que después de la transición. La percepción de la economía personal del votante posee materialmente el mismo impacto antes y después de la elección de 2000. Los resultados son contradictorios con la segunda hipótesis del presente estudio, lo cual sugiere que el fenómeno estudiado, la calificación que la ciudadanía otorga al Presidente, resulta complejo y debe considerar la inclusión de variables relacionadas con la percepción y con las condiciones objetivas que enmarcan dichos fenómenos. ${ }^{9}$

El impacto que tiene la identificación partidista en la calificación que el ciudadano le otorga al Presidente también fortalece, en cierto sentido, el argumento de que la ciudadanía tiene mayores expectativas sobre la competencia del Ejecutivo para atender problemas políticos y económicos después de la transición, razón por la cual los efectos de dichas variables son mayores después de 2000. Los modelos 1 y 2 muestran que los efectos de la identificación partidista son mayores antes de la transición de 2000 que después de ella. Tal hallazgo sugiere que el ciudadano antes de la alternancia "atendía" con mayor prontitud a su identificación partidista que a su preocupación sobre los problemas políticos y económicos de la nación. Esta variable es altamente significativa antes y después de 2000; sin embargo, el tamaño del coeficiente es mayor durante los últimos años de la etapa

9 Para lo cual se necesitan mejores datos, no disponibles por el momento, que permitan la inclusión de tales variables en un diseño de investigación futuro. El presente trabajo representa un esfuerzo para cubrir el "vacío" de un fenómeno poco estudiado y al ser una de las primeras investigaciones en su tipo,sus hallazgos deben difundirse para atender y buscar respuesta a un problema relevante en América Latina: la forma en que el crimen afecta la manera en que los ciudadanos califican a los líderes de las instituciones políticas que los gobiernan, en este caso al Presidente. 
priísta. La variable interactiva PRI X Identificación partidista del Modelo 3 ilustra que dicha diferencia es estadísticamente significativa.

\section{Conclusiones}

Desde la elección presidencial de 2000, en México el crimen ha ganado mayor importancia como tema de la agenda política nacional y por ende se ha vuelto más importante cuando el ciudadano califica al Presidente. Al mismo tiempo, las expectativas sobre la competencia del Ejecutivo para atender dicho problema se han fortalecido desde la transición.

Este estudio revela que la opinión del ciudadano sobre el crimen tiene mayores efectos en la calificación otorgada al Presidente después de la alternancia, que durante los últimos años del PRI en el poder. La presente investigación reconoce como limitante otra posible causal que pudiera explicar este cambio de comportamiento en la ciudadanía: la variación real del crimen en México. Contraargumento que en vez de descalificar el trabajo presentado complementaría los resultados obtenidos, de contar con dichos datos: la opinión de la ciudadanía sobre el Presidente semestral o trimestralmente, junto con la variación del crimen durante la misma unidad de tiempo.

Este mayor impacto de la percepción del crimen en la forma que la gente califica al presidente, coincide con un efecto más fuerte de la percepción del desempleo y el estado que conserva la economía del país. Lo cual sugiere que este aumento en las expectativas sobre la competencia del Presidente después de la transición, no sólo se enfoca en los temas político-sociales sino también en los económicos. En este último punto, resulta pertinente recordar que otras variables vinculadas con la percepción ciudadana de la inflación y la economía personal del votante tuvieron un comportamiento distinto. La primera tuvo mayores efectos antes de la elección de 2000, y la segunda, materialmente, tuvo los mismos efectos sobre la calificación del Presidente, antes y después de la transición. Lo cual sugiere que el fenómeno estudiado, la calificación que la ciudadanía le da al Ejecutivo, resulta complejo y debe considerar la inclusión de variables relacionadas con la percepción y con las condiciones objetivas que enmarcan dicho fenómeno, para lo cual se necesitan mejores datos que permitan la inclusión de tales variables en un diseño de investigación futuro.

Más allá del caso mexicano, este trabajo aporta elementos para el estudio de la aprobación presidencial en el área de la política comparada. Primero, el comportamiento político de los votantes no ocurre en un vacío. El contexto político afecta la manera en que la ciudadanía evalúa a las ins- 
tituciones de gobierno, en este caso del Poder Ejecutivo. Segundo, casos como el mexicano indican que nuestro conocimiento sobre los regímenes democráticos emergentes debe ser revaluado, para investigaciones futuras.

\section{Bibliografía}

Arce, Moisés, 2003, "Political Violence and Presidential Approval in Peru", The Journal of Politics, núm. 65, mayo, pp. 572-583.

Bermeo, Nancy, 1999, "Getting Mad or Going Mad: Citizens, Scarcity and the Break-down of Democracy in Interwar Europe", Working Paper, California, Center for the Study of Democracy/University of California, Irvine.

Banco de Información para la Investigación Aplicada en Ciencias Sociales (BIIACS), la encuesta se realizó el 12 de marzo de 1989, "Evaluación Nacional I", disponible en <http://biiacs-dspace.cide.edu/handle/10089/372>, consultado el 10 de febrero de 2011.

Banco de Información para la Investigación Aplicada en Ciencias Sociales (BIIACS), la encuesta se realizó el 1 de noviembre de 1994, “Encuesta Nacional. Evaluaciones: situación política, económica y social del país", disponible en: <http://biiacsdspace.cide.edu/handle/10089/450>, consultado el 5 de marzo de 2011.

Banco de Información para la Investigación Aplicada en Ciencias Sociales (BIIACS), la encuesta se realizó el 19 de julio de 1995, “Imagen del presidente", disponible en: <http://biiacs-dspace.cide.edu/handle/10089/3335>, consultado el 9 de enero de 2011.

Banco de Información para la Investigación Aplicada en Ciencias Sociales (BIIACS), la encuesta se realizó el 1 de noviembre de 1995, "Evaluación Nacional I", disponible en: <http://biiacs-dspace.cide.edu/handle/10089/3336>, consultado el 10 de enero de 2011.

Banco de Información para la Investigación Aplicada en Ciencias Sociales (BIIACS), la encuesta se realizó el 26 de enero de 2000, "Imagen del presidente Zedillo", disponible en: <http:/ / biiacs-dspace.cide.edu/handle/10089/1346>, consultado el 7 de enero de 2011.

Banco de Información para la Investigación Aplicada en Ciencias Sociales (BIIACS), la encuesta se realizó el 16 de marzo de 2001, “Situación política del país. Encuesta nacional de indicadores sobre diversos temas", disponible en: <http://biiacsdspace.cide.edu/handle/10089/13686>, consultado el 12 de febrero de 2011.

Banco de Información para la Investigación Aplicada en Ciencias Sociales (BIIACS), la encuesta se realizó el 6 de mayo de 2001, “Opinión sobre diversos actores políti- 
cos. Evaluación del presidente Fox y su gestión", disponible en: <http:/ / biiacsdspace.cide.edu/handle/10089/13664>, consultado el 10 de enero de 2011.

Banco de Información para la Investigación Aplicada en Ciencias Sociales (BIIACS), la encuesta se realizó el 23 de noviembre de 2001, "Opinión sobre diversos actores políticos. Evaluación del presidente Fox y su gestión", disponible en: <http://biiacs-dspace.cide.edu/handle/10089/13745>, consultado el 14 de enero de 2011.

Banco de Información para la Investigación Aplicada en Ciencias Sociales (BIIACS), la encuesta se realizó el 28 de noviembre de 2002, "Intención del voto. Imagen del presidente", disponible en: <http://biiacs-dspace.cide.edu/handle/10089/13744>, consultado el 28 de enero de 2011.

Banco de Información para la Investigación Aplicada en Ciencias Sociales (BIIACS), la encuesta se realizó el 27 de septiembre de 2003, “Situación política del país: evaluación del presidente Fox y su gestión", disponible en: <http:/ / biiacs-dspace.cide.edu/handle/10089/13732>, consultado el 4 de febrero de 2011.

Banco de Información para la Investigación Aplicada en Ciencias Sociales (BIIACS), la encuesta se realizó el 12 de junio de 2004, "Intención del voto: situación política del país: evaluación del presidente Fox y su gestión", disponible en: <http:/ / biiacs-dspace.cide.edu/handle/10089/13743>, consultado el 2 de febrero de 2011.

Baum, Christopher, 2006, An Introduction to Modern Econometrics Using Stata, Texas, Stata Press.

Buendía, Jorge, 1996, “Economic Reform, Public Opinion and Presidential Approval in Mexico 1988-1993", Comparative Political Studies, vol. 29, núm 5, pp. 566-591.

Camp, Roderic, 1999, "Democracy through Mexican Lenses", The Washington Quarterly, vol. 22, núm. 3, pp. 229-242

Camp, Roderic, 2004, “Citizen Attitudes toward Democracy and Vicente Fox's Victory in 2000", en Mexico's Pivotal Democratic Election, Stanford, California, Stanford University Press, pp. 25-46.

Clark, Mary, 2001, "Costa Rica: Portrait of an Established Democracy", en Citizen Views of Democracy in Latin America, Pittsburgh, University of Pittsburgh Press, pp. 73-90.

Cornelius, Wayne y Ann L. Craig, 1991, The Mexican Political System in Transition. Monograph Series 35, San Diego, California, Center for U.S.-Mexican Studies. 
Domínguez, Jorge I. y James McCann, 1995, “Shaping Mexico's Electoral Arena: The Construction of Partisan Cleavages in the 1988 and 1991 National Elections", en The American Political Science Review, México, vol. 89, no. 1, pp. 34-48.

Edwards, George C. III, William Mitchell y Reed Welch, 1995, “Explaining Presidential Approval: The Significance of Issue Salience," American Journal of Political Science 39, núm. 1, pp. 108-134.

Escalante, Gonzalbo Fernando, 2011, "Homicidios 2008-2009. La muerte tiene permiso", en Nexos, núm. 397, pp. 36-49.

Hagopian, Frances, 2005, “Conclusions: Government Performance, Political Representation, and Public Perceptions of Contemporary Democracy in Latin America", en The Third Wave of Democratization in Latin America, Cambridge, Cambridge University Press, pp. 319-362.

Instituto Nacional de Estadística Geografía e Informática (INEGI), 2012, Índice de Precios al Consumidor (base de datos), disponible en http:www.inegi.org.mx, consultado el 15 de enero de 2013.

Key, V. O, 1955, "A Theory of Critical Elections", The Journal of Politics, vol. 17, núm. 1, pp. 3-18.

Klesner, Joseph, 2001, “Legacies of Authoritarianism: Political Attitude in Chile and Mexico", en Citizen Views of Democracy in Latin America, Pittsburgh, University of Pittsburgh Press, pp. 118-138.

Magaloni, Beatriz, 2006, Voting for Autocracy, Cambridge, Cambridge University Press.

Magaloni, Beatriz y Alejandro Poiré, 2004, “The Issues, the Vote, and the Mandate for Change", en Mexico's Pivotal Democratic Election, Stanford, California, Stanford University Press, pp. 293-320.

Mainwaring, Scott y Aníbal Pérez-Liñán, 2005, “Latin America Democratization since 1978", en The Third Wave of Democratization in Latin America, Cambridge, Cambridge University Press, pp. 14-62.

Moreno, Alejandro, 2009, La decisión electoral. Votantes, partidos y democracia en México, ciudad de México, Miguel Ángel Porrúa.

Moreno, Alejandro, 2006, "Changing Ideological Dimension of Party Competition in Mexico, 1990-2006", presentado en la Annual Meeting of the American Political Science Association.

Moreno, Alejandro, 2004, "Destacan alternancia como mayor logro", en periódico Reforma, ciudad de México, 1 de diciembre, pp. 9-10. 
Moreno, Alejandro, 2001, "Democracy and Mass Belief Systems in America Latina", en Citizen Views of Democracy in Latin America, Pittsburgh, University of Pittsburgh Press, pp. 27-50.

Mishler, William y John P. Willerton, 2003, “The Dynamics of Presidential Popularity in Post-Communist Russia", The Journal of Politics, vol. 65, núm. 1, pp. 111-141.

Parametría, la encuesta se realizó en septiembre de 2006, "Encuesta Serie Nacional”, disponible en: <htto:/ /www.parametria.com. $m x>$, facilitada a solicitud expresa del autor, 20 de enero de 2011.

Parametría, la encuesta se realizó en noviembre de 2006, "Encuesta Serie Nacional”, disponible en: <htto:/ / www.parametria.com.mx>, facilitada a solicitud expresa del autor, 20 de enero de 2011.

Sánchez, Vicente, 2011, “La lucha actual del gobierno mexicano contra la delincuencia en la frontera con Estados Unidos", Frontera Norte, vol. 23, núm. 45, pp. 97130 .

Seligson, Mitchell, 2005, “Democracy on Ice: The Multiple Challenges of Guatemala's Peace Process", en The Third Wave of Democratization in Latin America, Cambridge, Cambridge University Press, pp. 202-234.

UNODC, 2011, Homicide Statistics, Dataset for Advanced Users, by Country and Source (2003-2008), disponible en <http:/ / www/ unodc.org/unodc/en/data-andanalysis/homicide.html>, consultado el 15 de mayo de 2011.

Villarreal, Andrés, 1999, "Public Opinion of the Economy and the President among Mexico City Residents: The Salinas Sexenio", Latin American Research Review, vol. 34, núm. 2, pp. 132-151.

Wood, Elisabeth, 2005, "Challenges to Political Democracy in El Salvador", en The Third Wave of Democratization in Latin America, Cambridge, Cambridge University Press, pp. 179-201.

Wooldridge, Jeffrey, 2001, Introducción a la econometría, ciudad de México, Thomson Learning.

Zhong, Yang Jie Chen y John Scheb, 1998, "Mass Political Culture in Beijing", Asian Survey, vol. 38, núm. 8, pp. 763-783.

Recibido el 9 de junio de 2011 Aceptado el 23 de enero de 2013 